\title{
Effect of Smoking on Retinal Thickness and Vascular Density in Thyroid Eye Disease
}

\author{
Mansooreh Jamshidian-Tehrani, Abolfazl Kasaei, Zahra Mahdizad, Masoud Aghsaei Fard, Mehdi Aminizade \\ Eye Research Center, Farabi Eye Hospital, Tehran University of Medical Sciences, Tehran, Iran
}

\begin{abstract}
Purpose: To evaluate the effect of smoking on retinal thickness and macular and peripapillary vascular density in thyroid eye disease (TED).

Methods: In this cross-sectional study, subjects diagnosed with TED were analyzed in three groups: smokers, passive smokers, and non-smokers. Ganglion cell complex thickness, total retinal thickness, macular superficial vascular plexus densities, deep vascular plexus densities, optic nerve head, and radial peripapillary capillary density were measured in each group.

Results: Twenty-two eyes (21.6\%) of active smokers, 11 eyes (10.8\%) of passive smokers, and 69 eyes (67.6\%) of non-smokers constitute the study subjects. Twenty-one eyes (12.6\%) had active status (clinical activity score $\geq 3$ ), 77 eyes (46.1\%) were neither active nor compressive, four eyes $(2.4 \%)$ of two patients constituted the compressive group. Age and disease activity adjusted analysis was performed. Ganglion cell complex thickness of smokers was significantly higher than non-smokers in the inferior hemi-parafoveal sector $(p=0.04)$. Active smokers had significantly higher $(p<0.01)$ retinal thickness in all sectors compared to non-smokers, except the foveal sector. Smokers had lower superficial vessel density in the superior parafoveal sector compared to non-smokers $(p=0.04)$. Considering deep vessel densities between smokers and non-smokers, no significant difference was observed. Radial peripapillary capillary densities (significant difference was observed in the whole image and infranasal peripapillary sector), Macular vascular densities (significant difference was observed in parafoveal sectors), and optic nerve head (not reaching statistical significance level in any sectors) were highest in passive smokers.
\end{abstract}

Conclusions: Smoking is associated with increased total retinal thickness. Macular vascular densities were not different between smokers and non-smokers in TED.

Key Words: Graves ophthalmopathy, Retinal diseases, Smoking

Thyroid eye disease (TED), also called "Graves' ophthalmopathy" is an autoimmune condition that is characteristi-

Received: April 15, 2021 Final revision: July 11, 2021

Accepted: July 12, 2021

Corresponding Author: Mehdi Aminizade, MD. Eye Research Center, Farabi Eye Hospital, Tehran University of Medical Sciences, Qazvin Square, Tehran 13366 16351, Iran. Tel: 98-912-068-8957, Fax: 98-66451063, E-mail: mehdiaminizade@yahoo.com cally seen in patients with hyperthyroidism. Common clinical features are those of extraocular tissues such as proptosis, strabismus, conjunctival and eyelid swelling, and redness, however, it can also become sight threatening [1,2]. Smoking as a major risk factor, poses a crucial role in the progression of TED [3]. Although the pathophysiologic mechanisms of TED are not fully comprehended, emerging technologies can help interpret new aspects of the disease process. Some diagnostic tests have been used such as visu- 
al field, visual evoked potential, contrast sensitivity test, and optical coherence tomography (OCT), to identify early structural changes and to prevent permanent visual loss [4].

Orbital hemodynamics and vascular abnormalities have been investigated in TED. Retrobulbar blood flow is abnormal in TED and superior ophthalmic vein (SOV) flow velocity is altered in active disease [5,6]. Reversed SOV blood flow and venous stasis have also been reported in dysthyroid optic neuropathy [7]. Smoking is believed to correlate with orbital venous congestion and lower minimum velocity in SOV flow in TED [8].

Optical coherence tomography angiography (OCTA) as an extension of OCT can quantify retinal and choroidal microvascular density [9]. Using OCTA, attenuation of peripapillary vascular density is disclosed in dysthyroid optic neuropathy [10]. Some other studies demonstrated a more prominent reduction in peripapillary and macular capillary densities in active TED $[4,11]$. On the other hand, there is a report of increased microvascular density in active TED [9]. This study aims to evaluate the effect of smoking on retinal thickness and macular and peripapillary vascular density in TED.

\section{Materials and Methods}

Patients with a confirmed diagnosis of TED who were referred Farabi Eye Hospital from July 2016 to September 2019 were recruited in the present study. This study was reviewed and approved by the ethical committee of Tehran University of Medical Sciences (IR.TUMS.FARABIH. REC.1396.4176). Informed consent for contribution in this study was obtained from all participants. Healthy subjects from patients' companions with normal ophthalmic examinations were registered as the control group. Patients with any kind of optic neuropathy such as glaucomatous optic neuropathy, diabetes mellitus associated and other retinopathies, idiopathic orbital inflammation, sarcoidosis, vasculitis, cellulitis, orbital tumors, systemic conditions such as hypertension, and those with refractive errors beyond \pm 6.00 diopters (D) or more than $3.00 \mathrm{D}$ of astigmatism were excluded [4].

Demographic data of all patients including age, gender, body mass index, blood pressure, smoking status (smoker, passive smoker, and non-smoker), and current systemic and topical medications were also gathered. Active smok- ing was defined as current daily smoking of at least one cigarette per day. Passive smoking was defined as environmental exposure to cigarette smoke by individuals other than active smokers. All participants underwent complete ophthalmic examinations. Best-corrected visual acuity, slit-lamp biomicroscopy, Hertel exophthalmometry, intraocular pressure (using Goldman applanation tonometry), fundus examination, and OCTA were evaluated for all patients. Laboratory serum biochemical parameters included thyroid-stimulating hormone, free thyroxine, free triiodothyronine, anti-thyroid-stimulating hormone receptor antibodies, anti-thyroid peroxidase antibodies, and anti-thyroglobulin antibodies. Diagnosis of TED was established according to Bartley's international diagnostic criteria [12]. Disease activity was classified according to clinical activity score (CAS). Two groups of patients were enrolled: patients with CAS $\geq 3$ at primary visit (active group) and patients with CAS $<3$ at primary visit (non-active non-compressive group) $[4,13,14]$.

All participants of the present study underwent macula and optic nerve head $(\mathrm{ONH})$ en-face OCTA imaging using RTVue XR Avanti Spectral Domain OCT system (Optovue, Fremont, CA, USA) with AngioVue software ver. 2015.1.0.26 [14,15]. To evaluate superficial and deep capillary plexuses a $6 \times 6-\mathrm{mm}$ macular scan was used. Additionally, parafoveal vascular density was measured in a 3-mm ring around the fovea. A $4.5 \times 4.5$-mm scan centered on the optic disc was utilized for ONH measurements. To assess radial peripapillary capillary (RPC) density, vascular densities between internal limiting membrane and posterior boundary of nerve fiber layer were captured. Total peripapillary vascular density was calculated as $\mathrm{ONH}$ image by recording vascular densities from internal limiting membrane to the retinal pigment epithelium. Vascular densities of both RPC and ONH images were also reported in six sections. Image quality was checked and images with a signal strength index of higher than 48 were included.

Descriptive statistics were calculated as mean and standard deviation. Linear mixed modeling, using Sidak correction for one-way ANOVA test, before and after adjustment for age and disease activity status, was employed for comparisons between groups and calculation of $p$-values. We used the chi-square test to evaluate categorical variables. IBM SPSS ver. 22.0 (IBM Corp., Armonk, NY, USA) was used to perform statistical analyses. Significant level was considered as $p$-values $<0.05$. 


\section{Results}

One hundred two eyes of 61 patients with TED were included in this study. fifty-seven patients $(93.4 \%)$ of 61 patients with TED had hyperthyroidism and only four patients $(6.6 \%)$ had hypothyroidism. Twenty-two eyes $(21.6 \%)$ of active smokers, 11 eyes (10.8\%) of passive smokers, and 69 eyes $(67.6 \%)$ of non-smokers constitute the study subjects. Mean age was $43.0 \pm 12.9$ years. Mean age of active smokers was $44.6 \pm 11.9$ years. Mean age of passive smokers and non-smokers was $40.1 \pm 14.2$ and $44.4 \pm 13$ years, respectively ( $p=0.42)$. In terms of disease activity, 21 eyes (12.6\%) had active status (CAS $\geq 3), 77$ eyes (46.1\%) were neither active nor compressive, four eyes (2.4\%) of two patients were in compressive status. Seventy-five point three percent (58 eyes) of neither active nor compressive group were non-smokers. Forty-seven point six percent (10 eyes) of the active group and 50\% (two eyes) of the compressive group were active smokers $(p=0.001)$. Mean intraocular pressure of active smokers, passive smokers, and nonsmokers were $17.7 \pm 2.5,15.8 \pm 1.2$, and $16.5 \pm 4.3$, respectively ( $p=0.4)$. Hertel exophthalmometry was performed and the results for active smokers, passive smokers, and non-smokers were $23.2 \pm 5.2,24.8 \pm 4.7$, and $22.3 \pm 4$, re- spectively $(p=0.31)$. Margin to reflex distance 1 was also measured and the results for active smokers, passive smokers, and non-smokers were $5.2 \pm 0.8,4.7 \pm 1.4$, and $5.1 \pm 1.1$, respectively ( $p=0.52$ ). Mean visual field mean deviation of active smokers, passive smokers and non-smokers were $-2.2 \pm 2.3,-1.4 \pm 2.5$, and $-1.3 \pm 2.2$, respectively $(p=0.2)$.

Ganglion cell complex (GCC) thickness was compared between smokers, passive smokers, and non-smokers. Although the mean GCC thickness was higher in smokers, the only statistically significant comparison was observed between smokers and non-smokers in the inferior hemiparafoveal sector $(p=0.04)$.

Total retinal thickness was also compared among the three groups. Active smokers had significantly higher $(p<$ $0.01)$ retinal thickness in all sectors compared to nonsmokers and passive smokers, except the foveal sector in which the difference between smokers and non-smokers was not statistically significant (Table 1).

Superficial macular vessel densities, measured by OCTA, were compared among three groups. Smokers had lower vessel density in the superior parafoveal sector compared to non-smokers $(p=0.04)$ (Table 2). Deep macular vessel densities were measured using OCTA. Comparing vessel densities between smokers and non-smokers, no sig-

Table 1. Macular and GCC thicknesses in the smoker, passive smoker, and non-smoker groups

\begin{tabular}{|c|c|c|c|c|c|c|}
\hline \multirow[b]{2}{*}{ Variable } & \multicolumn{3}{|c|}{ Smoking status } & \multicolumn{3}{|c|}{$p$-value ${ }^{*}$} \\
\hline & Smoker & Passive smoker & Non-smoker & $\begin{array}{c}\text { Smoker vs. } \\
\text { passive smoker }\end{array}$ & $\begin{array}{l}\text { Smoker vs. } \\
\text { non-smoker }\end{array}$ & $\begin{array}{c}\text { Passive } \\
\text { smoker vs. } \\
\text { non-smoker }\end{array}$ \\
\hline \multicolumn{7}{|l|}{ GCC thickness } \\
\hline Total parafovea & $125.5 \pm 10.8$ & $123.0 \pm 2.6$ & $120.6 \pm 11.6$ & 0.800 & 0.054 & 0.72 \\
\hline Superior hemi-parafovea & $124.3 \pm 11.5$ & $122.6 \pm 3.1$ & $120.4 \pm 11.3$ & 0.910 & 0.130 & 0.73 \\
\hline Inferior hemi-parafovea & $126.6 \pm 10.3$ & $123.2 \pm 3.1$ & $120.8 \pm 12.3$ & 0.670 & 0.040 & 0.78 \\
\hline \multicolumn{7}{|l|}{ Total retinal thickness } \\
\hline Fovea & $255.6 \pm 23.7$ & $236.2 \pm 12.5$ & $246.3 \pm 25.7$ & 0.010 & 0.090 & 0.21 \\
\hline Total parafovea & $326.1 \pm 17.1$ & $310.2 \pm 7.1$ & $312.3 \pm 16.3$ & 0.001 & $<0.001$ & 0.92 \\
\hline Superior hemi-parafovea & $326.5 \pm 17.7$ & $311.3 \pm 7.6$ & $313.1 \pm 16.4$ & 0.002 & $<0.001$ & 0.95 \\
\hline Inferior hemi-parafovea & $325.9 \pm 16.6$ & $309.1 \pm 7.7$ & $311.7 \pm 16.8$ & 0.001 & $<0.001$ & 0.87 \\
\hline Temporal parafovea & $316.1 \pm 17.4$ & $296.9 \pm 6.3$ & $304.1 \pm 18.4$ & 0.001 & 0.001 & 0.28 \\
\hline Superior parafovea & $329.6 \pm 16.9$ & $316.5 \pm 9.1$ & $317.0 \pm 16.0$ & 0.007 & $<0.001$ & 0.99 \\
\hline Nasal parafovea & $330.5 \pm 18.8$ & $314.9 \pm 9.8$ & $314.8 \pm 16.7$ & 0.002 & $<0.001$ & 1.00 \\
\hline Inferior parafovea & $328.4 \pm 16.8$ & $312.2 \pm 8.6$ & $313.8 \pm 16.7$ & 0.001 & $<0.001$ & 0.96 \\
\hline
\end{tabular}

Values are presented as mean \pm standard deviation.

$\mathrm{GCC}=$ ganglion cell complex.

*One-way ANOVA test was used to calculate $p$-values. 
Table 2. Superficial macular vascular densities in the smoker, passive smoker, and non-smoker groups

\begin{tabular}{|c|c|c|c|c|c|c|}
\hline \multirow[b]{2}{*}{ Variable } & \multicolumn{3}{|c|}{ Smoking status } & \multicolumn{3}{|c|}{$p$-value ${ }^{*}$} \\
\hline & Smoker & Passive smoker & Non-smoker & $\begin{array}{c}\text { Smoker vs. } \\
\text { passive smoker }\end{array}$ & $\begin{array}{l}\text { Smoker vs. } \\
\text { non-smoker }\end{array}$ & $\begin{array}{c}\text { Passive } \\
\text { smoker vs. } \\
\text { non-smoker }\end{array}$ \\
\hline Whole & $50.9 \pm 5.7$ & $53.4 \pm 2.0$ & $52.2 \pm 3.4$ & 0.09 & 0.23 & 0.57 \\
\hline Parafoveal & $53.3 \pm 5.9$ & $56.4 \pm 1.6$ & $54.6 \pm 3.7$ & 0.04 & 0.35 & 0.24 \\
\hline Superior hemi-parafoveal & $53.5 \pm 5.9$ & $56.5 \pm 1.4$ & $54.8 \pm 3.6$ & 0.03 & 0.27 & 0.25 \\
\hline Inferior hemi-parafoveal & $53.1 \pm 6.1$ & $56.4 \pm 2.1$ & $54.4 \pm 4.2$ & 0.08 & 0.49 & 0.31 \\
\hline Temporal parafoveal & $52.6 \pm 5.6$ & $55.4 \pm 1.8$ & $53.8 \pm 3.7$ & 0.05 & 0.31 & 0.31 \\
\hline Superior parafoveal & $53.5 \pm 6.5$ & $56.7 \pm 1.9$ & $55.8 \pm 3.8$ & 0.06 & 0.04 & 0.82 \\
\hline Nasal parafoveal & $53.2 \pm 5.5$ & $56.1 \pm 2.0$ & $53.8 \pm 4.3$ & 0.13 & 0.90 & 0.19 \\
\hline Inferior parafoveal & $53.3 \pm 6.9$ & $57.0 \pm 2.1$ & $54.8 \pm 5.0$ & 0.12 & 0.53 & 0.39 \\
\hline
\end{tabular}

Values are presented as mean \pm standard deviation.

*One-way ANOVA test was used to calculate $p$-values.

Table 3. Deep macular vascular densities in the smoker, passive smoker, and non-smoker groups

\begin{tabular}{|c|c|c|c|c|c|c|}
\hline \multirow[b]{2}{*}{ Variable } & \multicolumn{3}{|c|}{ Smoking status } & \multicolumn{3}{|c|}{$p$-value ${ }^{*}$} \\
\hline & Smoker & Passive smoker & Non-smoker & $\begin{array}{c}\text { Smoker vs. } \\
\text { passive smoker }\end{array}$ & $\begin{array}{l}\text { Smoker vs. } \\
\text { non-smoker }\end{array}$ & $\begin{array}{c}\text { Passive } \\
\text { smoker vs. } \\
\text { non-smoker }\end{array}$ \\
\hline Whole & $58.1 \pm 4.2$ & $60.3 \pm 1.6$ & $58.0 \pm 3.9$ & 0.15 & 1.0 & 0.056 \\
\hline Parafoveal & $60.8 \pm 4.0$ & $63.8 \pm 1.3$ & $60.9 \pm 1.3$ & 0.04 & 1.0 & 0.020 \\
\hline Superior hemi-parafoveal & $60.9 \pm 4.0$ & $64.0 \pm 1.6$ & $61.8 \pm 5.6$ & 0.20 & 0.8 & 0.400 \\
\hline Inferior hemi-parafoveal & $60.6 \pm 4.1$ & $63.5 \pm 1.4$ & $60.2 \pm 5.4$ & 0.17 & 1.0 & 0.053 \\
\hline Temporal parafoveal & $60.0 \pm 3.4$ & $62.9 \pm 2.0$ & $59.9 \pm 4.7$ & 0.04 & 1.0 & 0.020 \\
\hline Superior parafoveal & $61.9 \pm 4.7$ & $64.8 \pm 1.8$ & $62.7 \pm 4.0$ & 0.04 & 0.7 & 0.100 \\
\hline Nasal parafoveal & $60.0 \pm 3.9$ & $63.3 \pm 1.7$ & $60.2 \pm 5.3$ & 0.10 & 1.0 & 0.080 \\
\hline Inferior parafoveal & $61.2 \pm 4.8$ & $64.0 \pm 1.5$ & $60.7 \pm 6.4$ & 0.30 & 1.0 & 0.100 \\
\hline
\end{tabular}

Values are presented as mean \pm standard deviation.

*One-way ANOVA test was used to calculate $p$-values.

nificant difference was observed (Table 3). Mean vessel densities were highest in passive smokers in both superficial and deep macular vascular plexus (Table 2, 3).

ONH and RPC densities were evaluated among study groups. Whole image $\mathrm{ONH}$ vessel densities were $50.9 \pm 5.7$, $53.4 \pm 2$, and $52.2 \pm 3.4$ for active smokers, passive smokers, and non-smokers, respectively ( $p=0.19)$. Whole image RPC densities were $53.7 \pm 4.1,57.6 \pm 2.8$, and $55.5 \pm 3.8$ for active smokers, passive smokers, and non-smokers, respectively $(p=0.02)$ (Table 4).

\section{Discussion}

The effect of smoking on ocular blood circulation in TED has not been thoroughly investigated. This effect can be divided into two categories: the effect on orbital blood circulation and the effect on retinal microvasculature. Although, these two categories may not be two separate phenomena. Smoking is known to be associated with a decreased blood flow velocity of the SOV [16,17]. Some authors believe decreased SOV blood flow velocity is secondary to increased orbital muscle index [18]. Others didn't see any correlations between SOV blood flow velocity and muscle index [19]. Choroidal blood circulation is also al- 
Table 4. ONH and RPC densities in the smoker, passive smoker, and non-smoker groups

\begin{tabular}{|c|c|c|c|c|}
\hline \multirow{2}{*}{ Variable } & \multicolumn{3}{|c|}{ Smoking status } & \multirow{2}{*}{$p$-value } \\
\hline & Smoker & Passive smoker & Non-smoker & \\
\hline \multicolumn{5}{|l|}{$\mathrm{ONH}$} \\
\hline Whole image & $55.4 \pm 5.0$ & $58.1 \pm 2.6$ & $56.5 \pm 3.9$ & 0.19 \\
\hline Total peripapillary & $58.4 \pm 6.1$ & $61.5 \pm 2.5$ & $59.8 \pm 5.5$ & 0.27 \\
\hline Nasal peripapillary & $58.4 \pm 3.9$ & $60.9 \pm 2.4$ & $59.3 \pm 4.0$ & 0.22 \\
\hline Inferonasal peripapillary & $58.7 \pm 6.2$ & $62.8 \pm 3.5$ & $60.5 \pm 5.7$ & 0.15 \\
\hline Inferatemporal peripapillary & $62.8 \pm 5.7$ & $64.5 \pm 4.3$ & $63.6 \pm 4.0$ & 0.56 \\
\hline Superatemporal peripapillary & $60.7 \pm 4.5$ & $61.6 \pm 2.4$ & $60.9 \pm 5.8$ & 0.89 \\
\hline Superanasal peripapillary & $58.6 \pm 6.6$ & $60.7 \pm 6.3$ & $59.5 \pm 5.1$ & 0.09 \\
\hline Temporal peripapillary & $58.9 \pm 4.3$ & $60.7 \pm 4.1$ & $59.9 \pm 4.5$ & 0.57 \\
\hline \multicolumn{5}{|l|}{$\mathrm{RPC}$} \\
\hline Whole image & $53.7 \pm 4.1$ & $57.6 \pm 2.8$ & $55.5 \pm 3.8$ & 0.02 \\
\hline Total peripapillary & $60.4 \pm 4.2$ & $63.9 \pm 2.5$ & $62.1 \pm 4.3$ & 0.07 \\
\hline Nasal peripapillary & $59.4 \pm 4.1$ & $61.7 \pm 2.9$ & $60.0 \pm 4.4$ & 0.31 \\
\hline Inferanasal peripapillary & $59.1 \pm 7.4$ & $64.5 \pm 4.5$ & $62.1 \pm 5.5$ & 0.03 \\
\hline Inferatemporal peripapillary & $65.0 \pm 5.4$ & $67.9 \pm 4.4$ & $66.4 \pm 4.7$ & 0.23 \\
\hline Superatemporal peripapillary & $62.5 \pm 7.8$ & $65.4 \pm 1.8$ & $64.3 \pm 6.3$ & 0.39 \\
\hline Superanasal peripapillary & $58.9 \pm 7.0$ & $62.3 \pm 6.3$ & $60.5 \pm 5.8$ & 0.31 \\
\hline Temporal peripapillary & $61.6 \pm 4.6$ & $64.4 \pm 3.9$ & $63.1 \pm 5.5$ & 0.34 \\
\hline
\end{tabular}

Values are presented as mean \pm standard deviation.

$\mathrm{ONH}=$ optic nerve head; $\mathrm{RPC}=$ radial peripapillary capillary.

*One-way ANOVA test was used to calculate $p$-values.

tered after smoking [20]. Blood flow velocity of the central retinal artery and vein has been studied in TED. Sadeghi-Tari et al. [8] did not find any significant differences in color doppler parameters of central retinal artery and vein between smokers and non-smokers in TED.

Oxygen and nutrients supply to the human retina is provided via two sources. The outer retina is supplied by the blood circulation of the choriocapillaris. The inner half of the retina is supplied by branches of the central retinal artery [21]. These branches form superficial and deep vascular plexus in the retina, regarding the current OCT nomenclature. Superficial vascular plexus is located in the GCC (determined as nerve fiber layer, ganglion cell layer, and inner plexiform layer). The deep vascular plexus is segmented between the inner nuclear layer and the outer plexiform layer [22].

According to the results of the present study, total parafoveal GCC thickness was not significantly different between smokers and non-smokers in TED. Total retinal thickness of smokers was significantly higher than nonsmokers in all sectors except for the foveal sector. Consid- ering non-significant GCC thickness between smokers and non-smokers, the increased total retinal thickness in active smokers can be attributable to increased outer retinal thickness in this group. For example, the mean total parafoveal GCC thickness of the smoker group is 4.9 microns $(125.5-120.6=4.9)$ higher than non-smokers, and the mean total parafoveal retinal thickness of the smoker group is 13.8 microns $(326.1-312.3=13.8)$ higher than non-smokers. Accordingly, in the mentioned sector, 8.9 microns $(13.8-4.9=9.1)$ is the difference caused by retinal layers outer to GCC. This interpretation brings us to a hypothetical distinct effect of smoking on inner and outer retinal thickness. Another possible explanation is that as the total retinal thickness is the summation of all the retinal layers, any changes in the thickness of the retinal layers are more obvious when all the layers are put together (total retinal thickness). Sadeghi-Tari et al. [8] using color doppler imaging reported that cigarette smoking can be associated with orbital venous congestion. On the other hand, Tamaki et al. [20] using the laser speckle method, showed that smoking possibly can increase blood velocity 
in choroidal tissue. Increased retinal thickness, whether the outer retina or total retina, may be related to orbital venous congestion and altered choroidal blood circulation. Jamshidian Tehrani et al. [4] did not find any changes in retinal thickness in patients with TED. This contrast can be explained by the absence of the effect of smoking on subjects of that study. We recommend studies with higher sample sizes to examine the effect of smoking on inner, outer, and total retinal thickness in TED.

We also compared vascular densities of the superficial and deep macular vascular plexus. Although smokers generally had lower superficial vascular densities, there was no significant difference in superficial macular vascular densities between smokers and non-smokers. Deep macular vascular densities were not significantly different between smokers and non-smokers. Some recent studies demonstrated a dropout of macular retinal capillary densities in both superficial and deep retinal vascular plexus in TED [4,11]. Some other studies revealed macular vascular densities are not altered by smoking [21,23]. Consistently, our findings showed that superficial and deep macular vascular plexus densities are not altered by smoking in TED.

Surprisingly, superficial and deep vascular densities were highest in the passive smoker group. This difference was only notable in some sectors. To find a clear point of view with reasonable explanations, we suggest further studies with bigger study populations and more clear definitions of passive smoking. Comparing ONH densities among study groups revealed no significant differences. RPC densities were significantly higher in passive smokers in some comparisons.

There are several limitations in the current study. First, because of the cross-sectional nature of the present study and the absence of longitudinal data, we were not able to establish any causative correlations and we could not present any predictive effect of smoking on assessed parameters. Second, we divided study subjects into smokers, passive smokers, and non-smokers. Considering the exact number of cigarettes smoked per day and the duration of smoking (pack-year) could have helped us verify more reliable dose-dependent effects. Future studies with such considerations and also larger study sample sizes are essential to look into this matter.

In conclusion, results of this study demonstrate that smoking is associated with increased total retinal thickness in TED. Macular vascular densities were not different between smokers and non-smokers in TED. ONH, RPC, and macular vascular densities may be higher in passive smoking in TED.

\section{Conflict of Interest}

No potential conflict of interest relevant to this article was reported.

\section{References}

1. Weiler DL. Thyroid eye disease: a review. Clin Exp Optom 2017;100:20-5.

2. Lewis KT, Bullock JR, Drumright RT, et al. Changes in peripapillary blood vessel density in Graves' orbitopathy after orbital decompression surgery as measured by optical coherence tomography angiography. Orbit 2019;38:87-94.

3. Khong JJ, Finch S, De Silva C, et al. Risk factors for Graves' orbitopathy; the Australian thyroid-associated orbitopathy research (ATOR) study. J Clin Endocrinol Metab 2016;101:2711-20.

4. Jamshidian Tehrani M, Mahdizad Z, Kasaei A, Fard MA. Early macular and peripapillary vasculature dropout in active thyroid eye disease. Graefes Arch Clin Exp Ophthalmol 2019;257:2533-40.

5. Sadeghi-Tari A, Jamshidian-Tehrani M, Nabavi A, et al. Reply to: 'Comment on: effect of smoking on retrobulbar blood flow in thyroid eye disease'. Eye (Lond) 2017;31:814-5.

6. Jamshidian-Tehrani M, Nabavi A, Kasaee A, et al. Color Doppler imaging in thyroid eye disease and its correlation to disease activity. Orbit 2019;38:440-5.

7. Nakase Y, Osanai T, Yoshikawa K, Inoue Y. Color Doppler imaging of orbital venous flow in dysthyroid optic neuropathy. Jpn J Ophthalmol 1994;38:80-6.

8. Sadeghi-Tari A, Jamshidian-Tehrani M, Nabavi A, et al. Effect of smoking on retrobulbar blood flow in thyroid eye disease. Eye (Lond) 2016;30:1573-8.

9. Ye L, Zhou SS, Yang WL, et al. Retinal microvasculature alteration in active thyroid-associated ophthalmopathy. Endocr Pract 2018;24:658-67.

10. Zhang T, Xiao W, Ye H, et al. Peripapillary and macular vessel density in dysthyroid optic neuropathy: an optical coherence tomography angiography study. Invest Ophthal- 
mol Vis Sci 2019;60:1863-9.

11. Wu Y, Tu Y, Bao L, et al. Reduced retinal microvascular density related to activity status and serum antibodies in patients with Graves' ophthalmopathy. Curr Eye Res 2020;45:576-84.

12. Bartley GB, Gorman CA. Diagnostic criteria for Graves' ophthalmopathy. Am J Ophthalmol 1995;119:792-5.

13. Bartalena L, Baldeschi L, Dickinson A, et al. Consensus statement of the European Group on Graves' orbitopathy (EUGOGO) on management of GO. Eur J Endocrinol 2008;158:273-85.

14. Fard MA, Suwan Y, Moghimi S, et al. Pattern of peripapillary capillary density loss in ischemic optic neuropathy compared to that in primary open-angle glaucoma. PLoS One 2018;13:e0189237.

15. Fard MA, Jalili J, Sahraiyan A, et al. Optical coherence tomography angiography in optic disc swelling. Am J Ophthalmol 2018;191:116-23.

16. Tehrani MJ, Abtahi SM. Mini review on the effect of smoking on retrobulbar blood flow in thyroid eye disease. $J$ Clin Exp Opthamol 2018;9:750-2.

17. Monteiro ML, Angotti-Neto H, Benabou JE, Betinjane AJ. Color Doppler imaging of the superior ophthalmic vein in different clinical forms of Graves' orbitopathy. Jpn J Ophthalmol 2008;52:483-8.

18. Somer D, Ozkan SB, Ozdemir H, et al. Colour Doppler imaging of superior ophthalmic vein in thyroid-associated eye disease. Jpn J Ophthalmol 2002;46:341-5.

19. Alp MN, Ozgen A, Can I, et al. Colour Doppler imaging of the orbital vasculature in Graves' disease with computed tomographic correlation. Br J Ophthalmol 2000;84:1027-30.

20. Tamaki Y, Araie M, Nagahara M, Tomita K. Acute effects of cigarette smoking on tissue circulation in human optic nerve head and choroid-retina. Ophthalmology 1999; 106:564-9.

21. Ciesielski M, Rakowicz P, Stopa M. Immediate effects of smoking on optic nerve and macular perfusion measured by optical coherence tomography angiography. Sci Rep 2019;9:10161.

22. Campbell JP, Zhang M, Hwang TS, et al. Detailed vascular anatomy of the human retina by projection-resolved optical coherence tomography angiography. Sci Rep 2017;7:42201.

23. Ayhan Z, Kaya M, Ozturk T, et al. Evaluation of macular perfusion in healthy smokers by using optical coherence tomography angiography. Ophthalmic Surg Lasers Imaging Retina 2017;48:617-22. 